\title{
ASSESSMENT OF LIGHT POLLUTION IMPACT ON PROTECTED AREAS IN CHINA
}

\author{
Wei.Jiang a,b , Guojin. He ${ }^{\text {a,c,d, } * \text {,Yuan.Ni }}{ }^{\text {a,e }}$ \\ ${ }^{a}$ Institute of Remote Sensing and Digital Earth, Chinese Academy of Sciences, Beijing, China-jiangwei@radi.ac.cn \\ ${ }^{\mathrm{b}}$ University of the Chinese Academy of Sciences, Beijing, China \\ ${ }^{c}$ Key Laboratory of Earth Observation Hainan Province, Sanya, China-hegj@radi.ac.cn \\ ${ }^{\mathrm{d}}$ Sanya Institute of Remote Sensing, Sanya, China \\ ${ }^{\mathrm{e}}$ Key Laboratory of Spatial Data Mining \& Information Sharing of Ministry of Education, Spatial Information Research Center of Fu \\ jian Province, Fuzhou University, Fuzhou, China-269786073@qq.com
}

KEY WORDS: Night-time light, protected areas, light pollution, DMSP/OLS, China

\begin{abstract}
:
The Protected Areas (PAs) with natural, ecological and cultural value play important role in biological processes, biodiversity and ecosystem service. During the past years of rapid urban expansion in China, the spatial range and intensity of light pollution unprecedented increase. Historically, optical remote sensing and field survey data had been used to reveal that human activities impacted on PAs for individual areas and few papers documented the issue of light pollution impact on PAs at national scale. Here, time series night-time light satellite images of Defense Meteorological Satellite Program Operational Linescan System (DMSP-OLS) were selected to assess the light pollution impacted on PAs in China. The method we proposed can be effectively applied to assess the impact of light pollution on PAs and the percent of dark PAs decreased by 35.38\% from 1992 to 2012 at nationwide. The trend of light pollution of most PAs in stable, however, light pollution of the local area is increase significantly, especially in northern Xinjiang, Gansu, Xizang, Yunnan, Jiangsu and Shandong. Considering the current status of light pollution encroach into PAs, two strategies of appropriate buffer zone and wide measured for light pollution are also recommend.
\end{abstract}

\section{INTRODUCTION}

Protected Areas (PAs), which cover 12\% of Earth's land surface, play an important role in the biodiversity and ecosystem service and can contribute to species abundances and ecosystem functions(Loucks et al., 2008). With rapid social and economic development across worldwide, the extent and intensity of human activities are unprecedentedly expanding. Due to poor management and lack of monitoring, these activities have caused function degradation of PAs in local areas, thus, it severely threatens to the sustainable development of protected PAs(Gaston et al., 2015b). The impact of human activities on PAs is complexity and diversity. Among the various influencing factors, it is recognized that land-use change driven by urban expansion is a key factor affecting PAs (Shafiee et al., 2015). Light pollution is the comprehensive results of land-use change and socio-economic development (Gallaway et al., 2010). Particularly in the recent two decades, earth surface has been illuminated by nightlight at night. Light pollution has been universal environmental issue(Bennie et al., 2014). However, this issue is still not attracted wide attention. In the recent years, the impact of light pollution on ecosystem has been documented(Bennie et al., 2015), furthermore, some study also focus on the organismal level to discuss the influence on sleep(Raap et al., 2015), flight trajectory(Rodriguez et al., 2015), spawning(Mazor et al., 2013).

Compared with the traditional survey methods, remote sensing observation means is more efficient and low cost. With the rise of night-time light remote sensing, night-time light satellite image has been widely used in various fields, such as socioeconomic parameter simulation(Rybnikova and Portnov, 2014), urbanization process assessment(Liu et al., 2012), humanitarian assistance(Li et al., 2013a; $\mathrm{Li}$ and $\mathrm{Li}, 2014)$, ecological environment(Aubrecht et al., 2008). As well as, night-time light remote sensing also greatly enhances the light pollution exploration and it has become an indispensable technology for light pollution assessment(Bennie et al., 2014).

In the recent years, night-time light remote sensing has been used in light pollution assessment and the impact of light pollution on the ecosystem (Bennie et al., 2015; Gaston et al., 2015a). Defense Meteorological Satellite Program's Operational Linescan System(DMSP-OLS) night-time light image, landcover product and global ecosystem types were combined to assess light pollution exposure at global scale(Bennie et al., 2015). Gaston proposed a method to quantify the erosion of light pollution in the global protected areas system using calibration night-time light satellite data(Gaston et al., 2015a). The study suggested larger PAs were influenced by light pollution slightly, however, smaller PAs have experienced significant increase of light pollution. Furthermore, Davies assessed the light pollution exposure of marine protected areas and found that artificial light had increased across most marine protected areas(Davies et al., 2016).

As many globally important PAs located in China, it is urgent to assess the impact of light pollution on PAs. Additionally, evidence shows that PAs have been disturbed by human activities(Ebbensgaard, 2015). Therefore, assessment of light pollution impact on PAs in China is extremely needed from national scale. Based on advanced night-time light remote sensing technology and Geographic Information System (GIS) 
spatial analysis tools, the proposed assessment method first be applied to the Xilin Gol Natural Steppe Protected Area(XGNSPA), then, the characteristics of light pollution of PAs in China are described. Finally, the light pollution trend of PAs in china is discussed. In the discussion section, the cause and countermeasures are analysed.

\section{STUDY AREA}

China is one of the countries with abundant biodiversity in the world. Meanwhile, due to high-intensity human activities since the economic reform in 1978, China is one of the world's most threatened countries with biodiversity (Xu et al., 2017). In order to safeguard biodiversity and ecosystem function, China has established a large number of PAs. According to the UNEPWCMC report in 2016, 2158 PAs had been collected, covering $17.08 \%$ (1599.09 thousand $\mathrm{km}^{2}$ ) of the total terrestrial area and $3.77 \%$ (33.09 thousand $\mathrm{km}^{2}$ ) of total marine Area. The percentage of terrestrial PAs in China is higher than that of global (14.7\%), whereas, the percentage protected of marine is lower than that of global (10.1\%).The distribution of PAs is showed in Figure 1.

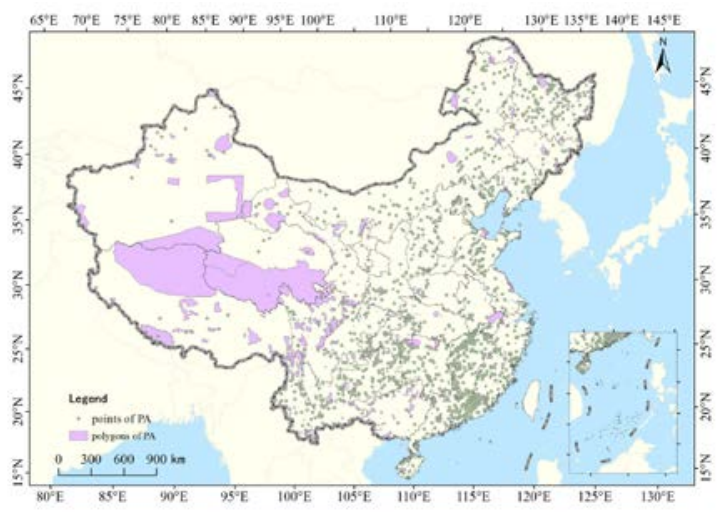

Figure 1. Study area with PAs positions

\section{DATAS}

\subsection{Night-time Light Imagery}

Currently, there are two commonly used night-time remote sensing data: Defense Meteorological Satellite Program's Operational Linescan System(DMSP-OLS) and Visible Infrared Imaging Radiometer Suite carried by the Suomi National PolarOrbiting Partnership (NPP-VIIRS)(Li et al., 2013b).Because DMSP-OLS is the longest time series night-time light satellite, it can be utilized to objectively demonstrate spatial and temporal evolution patterns of light pollution for a long time. Thus, version 4 DMSP-OLS night-time light images from 1992 to 2012 were selected, which can be downloaded from National Geophysical Data Center (NGDC) website of National Oceanic and Atmospheric Administration (NOAA).Among three DMSPOLS night-time light products, we selected cloud free coverage average stable night-time light product, which removed background noises, such as gas flaring, wildlife fire and aurora. This product has a 6bit radiometric resolution and the range of the digital number (DN) is 0 to 63 . The spatial resolution of this product is 30 arc second(about $1 \mathrm{k}$ around the equator).This product image of China in 2012 can be showed in Figure2.

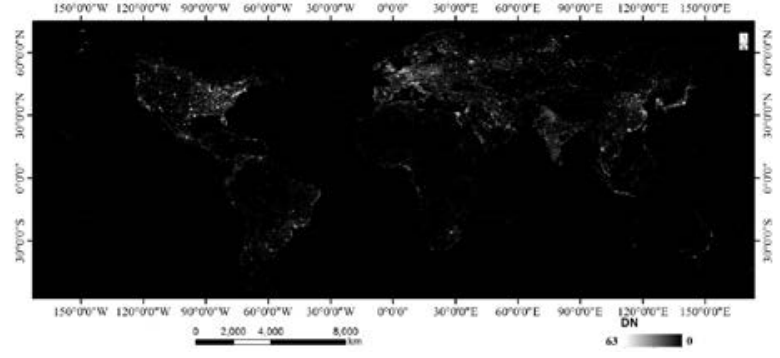

Figure 2. DMSP-OLS stable night-time light product of F182012

Because the systematic difference and degradation of sensor, evidence shows that there is significantly different of sensor at the same year(Jiang et al., 2017). Thus, in order to generate a consistent and comparable DMSP-OLS night-time light image, those images must be pre-processed. Jiang et al proposed a system process flow for time series DMSP-OLS night-time light image(Jiang et al., 2017), which includes three steps: model establishment, image correction and image post-processing. Through the system process flow, the temporal and spatial consistency of the image is improved greatly. Consequently, Images can be applied to light pollution assessment.

\subsection{Protected Areas Dataset}

With the support of the International Union for Conservation of Nature (IUCN), World Database on Protected Areas (WDPA) has been released by the United Nations Environment Programme's World Conservation Monitoring Centre (UNEPWCMC), which can be downloaded freely in public. After the release of the database, it has received the extensive concern by the global conservation biologists and been applied to evaluate the effectiveness of biodiversity conservation at global and regional scales (Davies et al., 2016; Gaston et al., 2015a). In addition to Hong Kong, Macao and Taiwan, the dataset contains a total of 2158 protected areas in China with boundary, area, protected level and establishment time information. In all of PAs, only 726 PAs have clear boundary, remaining PAs are the point type for the reason with smaller area. In this study, we concentrate on the impact of light pollution on clear boundary.

\section{METHODS}

\subsection{Night-time Light index}

In order to assess the light pollution characteristics in each PAs, we employed two easy and efficient night-time light indexes to demonstrate the level of light pollution(Jiang et al., 2017). The first is total night-time light (TNL) index and it represents the sum level of night-time pollution in individual PAs. TNL can be express as follows:

$$
T N L=\sum_{i=1}^{63} C_{i} \times D N_{i}
$$

where $D N_{i}$ is the $i$ th gray level, $C_{i}$ is the number of pixels that correspond to the gray level.

The second index is the night light mean (NTM). This index represents average level of light pollution in individual PAs and the formula is as follows:

$$
N T M=\left(\sum_{i=1}^{63} C_{i} \times D N_{i}\right) / \sum_{i=1}^{63} C_{i}
$$


where $D N_{i}$ is the $i$ th gray level, $C_{i}$ is the number of pixels that correspond to the gray level.

After construction of two night-time light indexes, the preprocessed DMSP-OLS night-time light image and PAs shape file were combined to automatic extract the index value using ArcGIS 10.2 software.

\subsection{Spatial Change Trend}

The night-time light change trend is important for us to learn about the history of influence of light pollution on PAs and make policy to control light pollution in PAs area. In this study, we adopt two method to analysis the night-time light change trend at pixel scale and PAs scale, which are namely mean composite difference (MCD)(Bennie et al., 2014) method and linear regression trend (LRT) method(Jiang et al., 2017).

Mean composite difference (MCD) method is proposed initially to explore the trend of light pollution across Europe. The formulas can be express as follow:

$$
\begin{array}{r}
M C D=\operatorname{mean}\left\{D N_{i}, D N_{i+1}, \ldots, D N_{i+n}\right\}- \\
\operatorname{mean}\left\{D N_{j}, D N_{j+1}, \ldots, D N_{j+m}\right\}
\end{array}
$$

where $D N_{i}, D N_{i+1} \ldots D N_{i+n}$ are represent first time series brightness value(BV) in $i$ th, $i+1$ th $\ldots i+n$th year at pixel scale, then the mean value of BV of each pixel can be calculated from $\mathrm{n}$ year. Similarly, the second time series BV $j$ th, $j+1$ th $\ldots j+m$ th year also can be calculated. Then, the difference of mean value at two periods can be used to demonstrate the trend of light pollution, and the positive of difference represents for the increase trend, whereas positive of difference represents decline trend.

What is more, linear regression trend method is employed to analyses the light pollution. Based on the calculated value in each PAs, the linear regression model can be established by the following formula,

$$
Y=\alpha * X+\varphi
$$

where $\mathrm{Y}$ is the TNL , $\mathrm{X}$ denotes the corresponding year, $\alpha$ is the slope and $\varphi$ is the intercept. Furthermore, the $\alpha$ can be represented by:

$$
\alpha=\frac{\sum_{i=1}^{n}\left(X_{i}-\bar{X}\right)\left(Y_{i}-\bar{Y}\right)}{\sum_{i=1}^{n}\left(X_{i}-\bar{X}\right)^{2}}
$$

If $\alpha>0$, it represents an increase trend of light pollution, if $\alpha$ $<0$, it represents a decline trend of light pollution.

\section{RESULTS}

\subsection{Case Study}

In order to show how the method assesses the impact of light pollution on PAs, the Xilin Gol Natural Steppe Protected Area(XGNSPA) was selected as case study. To begin with, time series DMSP-OLS night-time light images from 1992-1995 and 2009-2012 are used to mean composite and the result can be show Figure3 (a) and Figure3 (b).Compared with two mean composite results, we can clearly find that the density and extent of light pollution expand significantly. Furthermore, MCD method is adopted to quantify the extension and the increase trend area of light pollution mainly locates in southwest of PAs. According to the GF-1 $16 \mathrm{~m}$ resolution image (Figure4), the light pollution increase is caused by urban mine exploration in PAs. It also should note that the light pollution remains at a low level in addition to the mine area.

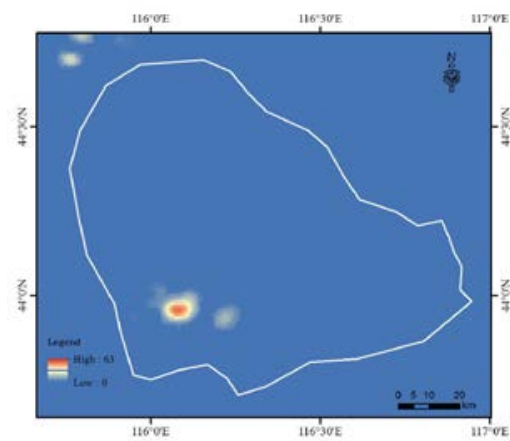

(a)

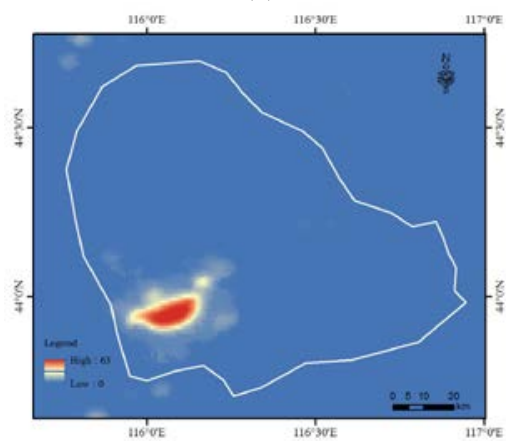

(b)

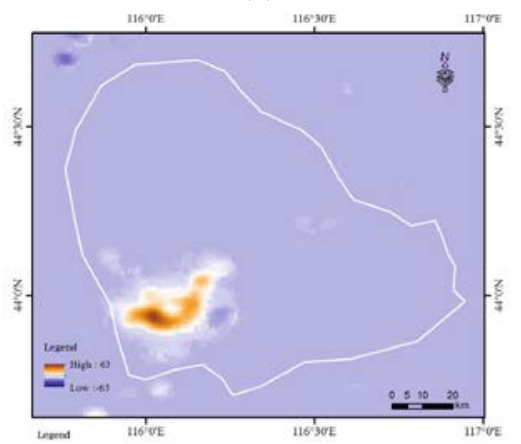

(c)

Figure 3. The trend of light pollution in XGNSPA (night-time light mean value in 1992-1995(a) and 2009-2012(b), the calculated change trend using MCD method)

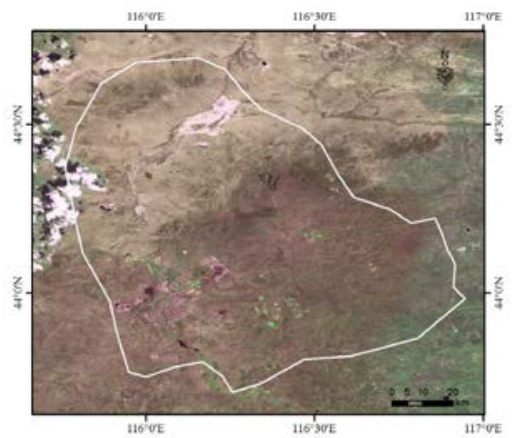

Figure 4. The GF-1 WFV image of XGNSPA

Moreover, the night-time light index method is adopted to quantitative analysis change of light pollution in PAs scale and the result is showed as Figure5.The TNL is showing increase trend with a fast growth rate, $449 \%$ and the slope of TNL is 739.77.Moreover, NMT increases from 0.34 to 
1.85.Consequently, From the quantitative analysis of the index method, we can find that the light pollution increase significantly.

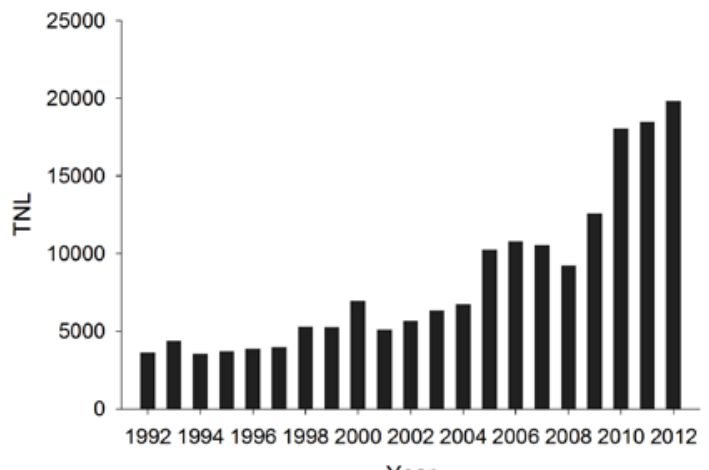

(a)

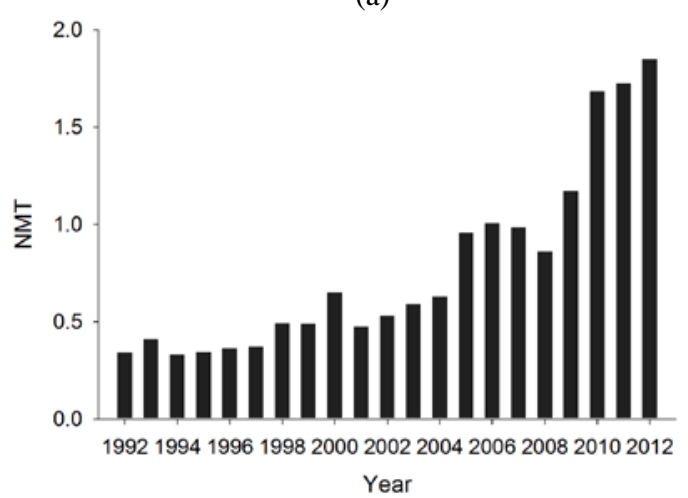

(b)

Figure 5. The night-time light index ((a)TNL and (b)NMT)of XGNSPA from 1992-2012

Combined with trend analysis and quantitative index analysis, the significant trend of light pollution in XGNSPA can be determined. From the perspective of light pollution spatial pattern, it mainly locates in the Xinlin Gol urban area and the rest of PAs is still in the dark at night. This result may demonstrate that the impacts of light pollution on PAs may concentrate in local area with high human activity intensity and the remaining regional luminous pollution effect is very slight.

\subsection{Light Pollution Characteristics of PAs in China}

Depending on the boundary of PAs, the TNL index was calculated automatically for each patch. Figure5a and Figure5b showed the TNL index of PAs in 1992 and 2012 across national scale, respectively. In 1992, 455 PAs were under dark (TNL=0), accounting for $62.59 \%$ of the total. Whereas, the 294 PAs are under dark $(\mathrm{TNL}=0)$. These PAs include 281 stable dark PAs and 13 decline light pollution PAs. Compared with the result of 1992, the percent of dark PAs decreased by $35.38 \%$.

From the perspective of spatial pattern PAs, the low light pollution effects of PAs mainly located at west and northeast region in 1992, whereas, some of western PAs have been lit in 2012. Moreover, most high light pollution effects of PAs located at east and central region in 1992 and 2012, and some PAs located in west region have been lit. Therefore, this result demonstrated that the impact of light pollution on the west region is strengthening.

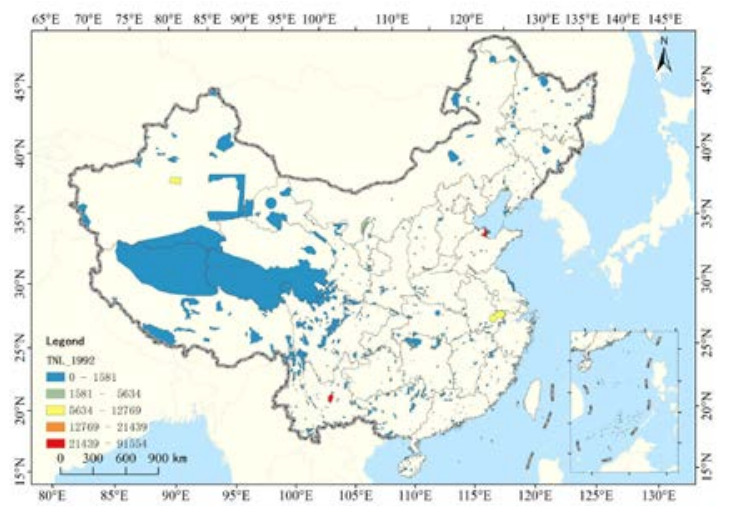

(a)

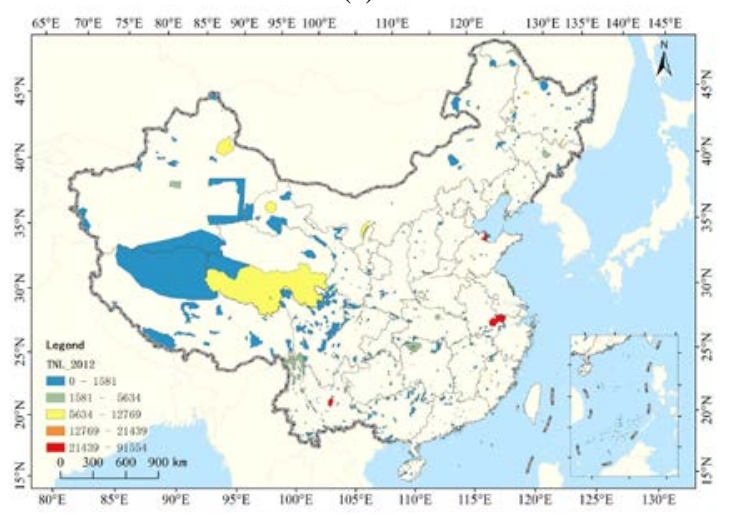

(b)

Figure 6. The TNL index of PAs across the national scale ((a) 1992 and (b) 2012)

\subsection{Light Pollution Trend of PAs in China}

For the purpose of quantifying the change trend of light pollution in PAs at national scale, the linear regression trend method was applied to identify for each PAs (figure7). Most PAs are belong to decrease (-518.42) and stable (-518.42-31.46) type, however, light pollution of some PAs located in northern Xinjiang, Gansu Xizang, Yunnan, Jiangsu and Shandong are showing significant increase. Generally, the pattern of light pollution impact on PAs are balanced from west China to east China and some PAs, located in local area, such as Dianchi, Yangtze river and Yellow river, are severally impacted by light pollution. This is attributable to these PAs are extremely close to human settlement. During the process of urban expansion over the past two decades, light pollution expanded significantly around human settlement, therefore, the PAs closing to cities show an increase trend.

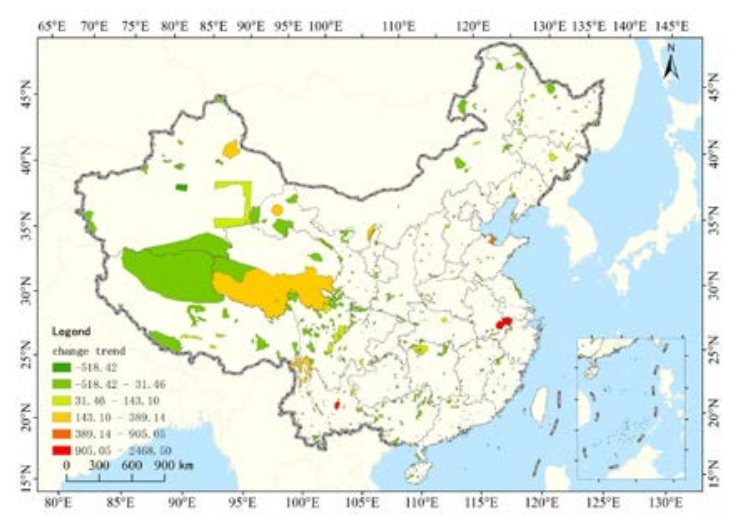

Figure 7. The change trend of TNL index of PAs across the national scale from 1992 to 2012 


\section{DISCUSSION}

With rapid City expansion and huge energy consumption around the world, light pollution has been a vital environmental topic for environmental scientists and policy makers. PAs are are great important for maintaining sustainable development of human society and nature(Loucks et al., 2008). In the recent year, the issue about light pollution influenced on PAs has raised widespread attention (Bennie et al., 2014; Falchi et al., 2016). Generally, ecological protection and economic development cannot be balanced, especially in fast developing countries, such as China. The initial idea of this study is to assess the impact of light pollution on PAs over the past two decades in China. Based on the analysis result, the impact of light pollution on the PAs is strengthening in the western region and the PAs change trend of light pollution in western China and east China are increasing. In the east China region, these increasing trend mainly relates to urban extent and the distance between PAs and city is a factor that cannot be ignored. Commonly, night time light of the city can be scattered into the sky(Cinzano and Falchi, 2014), thus, PAs will be lit up around the city. Similarly, light pollution of PAs increased in western China region mainly relates to the western development strategy. Despite the intensity of human activity is low, the continuous policy support accelerated the process of light pollution and the increase rate of western China is higher than east China. Moreover, electricity popularity in western China is also the positive factor.

Based on the trend and cause of light pollution in PAs, the two strategy to limit the impact of light pollution should be recommended. First the buffer zone between PAs and city region should be set up, especially in high influence PAs region. Second, the more measured for light pollution in PAs should be taken across nationwide integrate ecosystem service and landscape ecology knowledge. Moreover, this measure can be invoked as a basis for setting PAs in the further.

\section{CONCLUSIONS}

In this paper, time series corrected DMSP-OLS night-time light images from 1992-2012 was used to assess the impact of light pollution on PAs in China. First, the quantitatively assessment method, including night-time light index and spatial change trend, was established. Second, the XGNSPA was selected as a case study to test the assessment method. Finally, characteristics and trend of light pollution of PAs in China were analysed at national scale. The conclusion of this study is as follows:

(1) Based the XGNSPA case study, the method established in this study can be effectively applied to assess the impact of light pollution on PAs. Mine exploration is the main reason for the rapid growth of light pollution in XGNSPA.

(2) Compared with TNL of PAs in 1992 and 2012 across national wide, the percent of dark PAs decreased by $35.38 \%$ and the light pollution in PAs of western region is strengthening.

(3) Through the quantitative change trend method, the long time series (1992 to 2012) impact of light pollution on PAs were identified. The trend of light pollution in most PAs is stable, whereas some PAs locate in northern Xinjiang, Gansu Xizang, Yunnan, Jiangsu and Shandong are increasing significantly.
Based the result of this study, the urban extension driven by rapid economic development and the distance between PAs and city area contribute the increasing trend of light pollution in PAs, hence , the two strategy ,including ecological buffer zone and measured for light pollution are proposed to alleviate the trend. The conclusion can help the policy makers regulate the light pollution in PAs and maintain function and sustainable development of ecosystems in PAs.

\section{ACKNOWLEDGEMENTS}

This research was financially supported by The National Key Research and Development Program of China-rapid production method of large scale global change products (2016YFA0600302), the Hainan Provincial Department of Science and Technology under Grant Nos. ZDKJ2016021 and ZDKJ2016015.

\section{REFERENCES}

Aubrecht, C., Elvidge, C.D., Longcore, T., Rich, C., Safran, J., Strong, A.E., Eakin, C.M., Baugh, K.E., Tuttle, B.T., Howard, A.T. and Erwin, E.H., 2008. A global inventory of coral reef stressors based on satellite observed night-time lights. Geocarto International,23(6), 467-479.

Bennie, J., Davies, T.W., Duffy, J.P., Inger, R. and Gaston, K.J., 2014. Contrasting trends in light pollution across Europe based on satellite observed night time lights. Scientific Reports,4(

Bennie, J., Duffy, J.P., Davies, T.W., Correa-Cano, M.E. and Gaston, K.J., 2015. Global Trends in Exposure to Light Pollution in Natural Terrestrial Ecosystems. Remote Sensing,7(3), 2715-2730.

Cinzano, P. and Falchi, F., 2014. Quantifying light pollution. Journal of Quantitative Spectroscopy \& Radiative Transfer,139(13-20.

Davies, T.W., Duffy, J.P., Bennie, J. and Gaston, K.J., 2016. Stemming the Tide of Light Pollution Encroaching into Marine Protected Areas. Conservation Letters,9(3), 164-171.

Ebbensgaard, C.L., 2015. Urban Lighting, Light Pollution and Society. European Planning Studies,23(7), 1437-1440.

Falchi, F., Cinzano, P., Duriscoe, D., Kyba, C.C.M., Elvidge, C.D., Baugh, K., Portnov, B.A., Rybnikova, N.A. and Furgoni, R., 2016. The new world atlas of artificial night sky brightness. Science Advances,2(6).

Gallaway, T., Olsen, R.N. and Mitchell, D.M., 2010. The economics of global light pollution. Ecological Economics,69(3), 658-665.

Gaston, K.J., Duffy, J.P. and Bennie, J., 2015a. Quantifying the erosion of natural darkness in the global protected area system. Conservation Biology,29(4), 1132-1141.

Gaston, K.J., Visser, M.E. and Hoelker, F., 2015b. The biological impacts of artificial light at night: the research challenge. Philosophical Transactions of the Royal Society BBiological Sciences,370(1667).

Jiang, W., He, G., Long, T., Wang, C., Ni, Y. and Ma, R., 2017. Assessing Light Pollution in China Based on Night-time Light Imagery. Remote Sensing.

Li, X., Chen, F. and Chen, X., 2013a. Satellite-Observed Nighttime Light Variation as Evidence for Global Armed Conflicts. Ieee Journal of Selected Topics in Applied Earth Observations and Remote Sensing,6(5), 2302-2315.

$\mathrm{Li}, \mathrm{X}$. and Li, D., 2014. Can night-time light images play a role in evaluating the Syrian Crisis? International Journal of Remote Sensing,35(18), 6648-6661. 
Li, X., Xu, H.M., Chen, X.L. and Li, C., 2013b. Potential of NPP-VIIRS Night-time Light Imagery for Modeling the Regional Economy of China. Remote Sensing,5(6), 3057-3081. Liu, Z.F., He, C.Y., Zhang, Q.F., Huang, Q.X. and Yang, Y., 2012. Extracting the dynamics of urban expansion in China using DMSP-OLS night-time light data from 1992 to 2008. Landscape and Urban Planning,106(1), 62-72.

Loucks, C., Ricketts, T.H., Naidoo, R., Lamoreux, J. and Hoekstra, J., 2008. Explaining the global pattern of protected area coverage: relative importance of vertebrate biodiversity, human activities and agricultural suitability. Journal of Biogeography,35(8), 1337-1348.

Mazor, T., Levin, N., Possingham, H.P., Levy, Y., Rocchini, D., Richardson, A.J. and Kark, S., 2013. Can satellite-based night lights be used for conservation? The case of nesting sea turtles in the Mediterranean. Biological Conservation,159(63-72.

Raap, T., Pinxten, R. and Eens, M., 2015. Light pollution disrupts sleep in free-living animals. Scientific Reports,5(

Rodriguez, A., Rodriguez, B. and Negro, J.J., 2015. GPS tracking for mapping seabird mortality induced by light pollution. Scientific Reports,5(

Rybnikova, N.A. and Portnov, B.A., 2014. Mapping geographical concentrations of economic activities in Europe using light at night (LAN) satellite data. International Journal of Remote Sensing,35(22), 7706-7725.

Shafiee, M., Saffarian, S. and Zaredar, N., 2015. Risk Assessment of Human Activities on Protected Areas: A Case Study. Human and Ecological Risk Assessment,21(6), 14621478.

Xu, W.H., Xiao, Y., Zhang, J.J., Yang, W., Zhang, L., Hull, V., Wang, Z., Zheng, H., Liu, J.G., Polasky, S., Jiang, L., Xiao, Y., Shi, X.W., Rao, E.M., Lu, F., Wang, X.K., Daily, G.C. and Ouyang, Z.Y., 2017. Strengthening protected areas for biodiversity and ecosystem services in China. Proceedings of the National Academy of Sciences of the United States of America,114(7), 1601-1606. 